Applied Mathematical Sciences, Vol. 9, 2015, no. 12, 583 - 592

HIKARI Ltd, www.m-hikari.com

http://dx.doi.org/10.12988/ams.2015.411900

\title{
On Powers of General Tridiagonal Matrices
}

\author{
Qassem M. Al-Hassan \\ Department of Mathematics \\ Yarmouk University, Irbid, Jordan
}

Copyright (C) 2014 Qassem M. Al-Hassan. This is an open access article distributed under the Creative Commons Attribution License, which permits unrestricted use, distribution, and reproduction in any medium, provided the original work is properly cited.

\begin{abstract}
In this paper, a method for calculating powers of general tridiagonal matrices is introduced. This method employs the close relationship among tridiagonal matrices, second-order linear homogeneous difference equations, and orthogonal polynomials. Some examples are included to demonstrate the implementation of the method.
\end{abstract}

Mathematics Subject Classification: 65Q05; 39A05

Keywords: difference equations; tridiagonal matrices; orthogonal polynomials; powers and inverses

\section{Introduction}

It is well known that tridiagonal matrices have been under focus of many researchers recently. This emerged from the fact that such matrices play important roles in many recent applications, such as boundary value problems, parallel computing, spline interpolation, numerical solution of ordinary and partial differential equations, telecommunication system analysis. The calculation of powers- positive and/or negative- of tridiagonal matrices is therefore needed in order to solve problems that arise in these important applications. Consequently, a quite large number of publications that address this subject appeared in recent years. Some of these publications discuss inversion of these matrices in general, or specially structured types of them $[1,2,4$, 
$5,6,7,10,11]$, some other publications discuss powers of such specially structured matrices $[8,9,12,13,14,15]$, and some discuss both inversion and powers of these matrices [3].

This present work employs the close relationship among tridiagonal matrices, second- order linear homogeneous difference equations, and orthogonal polynomials, in order to derive an algorithm that does the job in computing powers of tridiagonal matrices with real or complex entries.

Given a general tridiagonal matrix with real or complex entries, we start by converting this matrix into a symmetric tridiagonal matrix. The case of a tridiagonal matrix with nonnegative real entries, which results in a real symmetric matrix, was discussed in detail in [2]. But the case of a general tridiagonal matrix with entries that can be real- both positive and/or negativeor possibly complex may result in a symmetric complex or a Hermitian matrix. This work addresses these two cases, and gives two different schemes that compute powers of the complex symmetric matrices.

This work parallels the line of [2] in the sense that a second- order linear homogeneous difference equation is used in order to generate a set of orthogonal polynomials of degree $\mathrm{n}$ when the matrix has size $n x n$. It is known that each polynomial of degree $i, i=1, \ldots, n$, is in fact the the determinant of the principal $i x i$ submatrix of the $n x n$ tridiagonal matrix given by:

$$
\left[\begin{array}{ccccc}
\frac{x-a_{1}}{b_{1}} & 1 & 0 & \ldots & 0 \\
\frac{c_{2}}{b_{2}} & \frac{x-a_{2}}{b_{2}} & 1 & 0 \ldots & 0 \\
0 & \frac{c_{3}}{b_{3}} & \frac{x-a_{3}}{b_{3}} & 1 \ldots & 0 \\
\cdots & \cdots & \cdots & \ldots & \ldots \\
& & 0 & \frac{c_{n}}{b_{n}} & \frac{x-a_{n}}{b_{n}}
\end{array}\right]
$$

The above mentioned difference equation has the form:

$$
p_{n}(x)=\frac{x-a_{n}}{b_{n}} p_{n-1}(x)-\frac{c_{n}}{b_{n}} p_{n-2(x),}, n \geq 2
$$

with initial conditions: $p_{0}(x)=1, p_{1}(x)=\frac{x-a_{1}}{b_{1}}$, where $a_{n} \neq 0, b_{n} \neq 0, c_{n} \neq 0$, for all $n \geq 0$,and $c_{1}=b_{n}=1$, and it defines the recursion relation for the set of orthogonal polynomials $\left\{p_{n}(x)\right\}_{n \geq 0}$ on an open interval $I$ with respect to a nonnegative weight function $w(t)$.

The set of orthogonal polynomials $\left\{p_{0}, p_{1}, \ldots, p_{n}\right\}$ plays an essential role in the construction of the eigenvalues and their corresponding eigenvectors of the matrix:

$$
\mathbf{T}=\left[\begin{array}{ccccc}
a_{1} & b_{1} & 0 & \ldots & 0 \\
c_{2} & a_{2} & b_{2} & 0 & \\
0 & c_{3} & a_{3} & b_{3} & \\
\ldots & \ldots & \ldots & \ldots & \ldots \\
& & & c_{n} & a_{n}
\end{array}\right]
$$


This matrix $T$ is converted by a similarity transformation into a symmetric real matrix, or a symmetric complex matrix, or a Hermitian matrix, we denote this matrix by $J$.

In the cases of a real symmetric matrix, or a Hermitian matrix, we construct the eigendecomposition of the matrix, which in turn is used to compute different powers of the matrix. In the case of a complex symmetric matrix, it is known that such matrices do not have the same properties of the other previous two cases of having an eigendecomposition that can be easily computed, therefore we have to use a different type of factorization, called Takagi factorization, which is then used to compute powers of the complex symmetric matrix.

This paper is organized as follows: Section 2 contains preliminary mathematical background needed for this work, section 3 contains the main results of the paper, this section is divided into three subsections, the first discusses the case of real symmetric matrices, the second discusses the case of Hermitian matrices, and the third contains the case of complex symmetric matrices, and section 4 presents some examples that demonstrate the method we introduce in this article about computing powers of matrices.

\section{Preliminaries}

Tridiagonal matrices, orthogonal polynomials, and second-order linear homogeneous difference equations are very much related with each other. Equation (2) above defines the recursion relation that generates a set of orthogonal polynomials $\left\{p_{n}(x)\right\}_{n \succeq 0}$ on an interval $I$ with respect to a nonnegative weight function $w(x)$. This is also related with tridiagonal matrices $(1)$ in the sense that $p_{n}(x)$ is equal to the determinant of the tridiagonal matrix in (1) above [4].

Equation (2) can be rewritten as:

$$
\frac{x}{b_{n}} p_{n-1}(x)=\frac{a_{n}}{b_{n}} p_{n-1}(x)+\frac{c_{n}}{b_{n}} p_{n-2}(x)+p_{n}(x)
$$

which in turn can be rewritten in the following matrix form:

$$
\mathbf{x .}\left[\begin{array}{ccccc}
\frac{1}{b_{1}} & 0 & & & 0 \\
0 & \frac{1}{b_{2}} & 0 & & 0 \\
0 & 0 & \frac{1}{b_{3}} & 0 & \\
& \ldots & \cdots & \ldots & \ldots \\
& & & 0 & \frac{1}{b_{n}}
\end{array}\right]\left[\begin{array}{c}
p_{0}(x) \\
p_{1}(x) \\
\cdot \\
\cdot \\
p_{n-1}(x)
\end{array}\right]=\left[\begin{array}{ccccc}
\frac{a_{1}}{b_{1}} & 1 & 0 & \ldots & 0 \\
\frac{c_{2}}{b_{2}} & \frac{a_{2}}{b_{2}} & 1 & 0 \ldots & 0 \\
0 & \frac{c_{3}}{b_{3}} & \frac{a_{3}}{b_{3}} & 1 \ldots & 0 \\
\ldots & \cdots & \cdots & \ldots & \ldots \\
& & 0 & \frac{c_{n}}{b_{n}} & \frac{a_{n}}{b_{n}}
\end{array}\right]\left[\begin{array}{c}
p_{0}(x) \\
p_{1}(x) \\
\cdot \\
\cdot \\
p_{n-1}(x)
\end{array}\right]+\left[\begin{array}{c}
0 \\
0 \\
\cdot \\
\cdot \\
p_{n}(x)
\end{array}\right]
$$

or

$$
\mathbf{x} \cdot \mathbf{D} \cdot \mathbf{p}=\mathbf{A} \cdot \mathbf{p}+\mathbf{e}_{n}^{T} \cdot p_{n}(x)
$$

where $\mathbf{D}=\operatorname{Diag}\left(\frac{1}{b_{1}}, \frac{1}{b_{2}}, \ldots, \frac{1}{b_{n}}\right)$, and $\mathbf{p}=\left[p_{0}(x), p_{1}(x), \ldots, p_{n-1}(x)\right]^{T}, A=\left[\begin{array}{cccccc}\frac{a_{1}}{b_{1}} & 1 & 0 & \ldots & 0 \\ \frac{c_{2}}{b_{2}} & \frac{a_{2}}{b_{2}} & 1 & 0 \ldots & 0 \\ 0 & \frac{c_{3}}{b_{3}} & \frac{a_{3}}{b_{3}} & 1 \ldots & 0 \\ \ldots & \ldots & \ldots & \ldots & \ldots \\ & & 0 & \frac{c_{n}}{b_{n}} & \frac{a_{n}}{b_{n}}\end{array}\right]$, and 
$\mathbf{e}_{n}=[0,0, \ldots, 1]^{T} \in R^{n}$.

It is also known that $\left\{p_{n}(x)\right\}_{n \succeq 0}$ is a set of orthogonal polynomials on an interval $I$ with respect to a weight function $w(x)$, each polynomial $p_{j}(x), 0 \leq j \leq n$ has $j$ distinct real roots in the interior of the interval $I$.

So, if $x$ is replaced by $x_{j}$ in(4), where $x_{j}$ is a root of $p_{n}(x)$, then (4) becomes:

$$
x_{j} \cdot D \cdot p\left(x_{j}\right)=A \cdot p\left(x_{j}\right)
$$

Which implies that $x_{j}, j=1, \ldots, n$,is a solution of the generalized eigevalue problem: $\lambda D u=$ $A u$, which is equivalent to $\left(D^{-1} A\right) u=\lambda u$, where $D^{-1} A$ is the tridiagonal matrix:

$$
\mathbf{D}^{-1} \mathbf{A}=\mathbf{T}=\left[\begin{array}{ccccc}
a_{1} & b_{1} & 0 & \ldots & 0 \\
c_{2} & a_{2} & b_{2} & 0 & \\
0 & c_{3} & a_{3} & b_{3} & \\
\cdots & \cdots & \cdots & \ldots & \cdots \\
& & & c_{n} & a_{n}
\end{array}\right]
$$

Therefore, $x_{j}, j=1, \ldots, n$, are the eigenvalues of the tridiagonal matrix $T$, and the vector $\left[p_{0}\left(x_{j}\right), p_{1}\left(x_{j}\right), \ldots, p_{n-1}\left(x_{j}\right)\right]^{T}$ is the corresponding eigenvector. Moreover, the matrix $\mathrm{B}$ whose columns are the eigenvectors of $T$ is nonsingular, because the matrix $\mathbf{A}$ is also nonsingular, and consequently, the eigenvectors are linearly independent.

\section{Main Results}

Let a tridiagonal matrix with real or complex entries be given, then this matrix can be transformed using a similarity transformation into one of the following:

1. A real symmetric matrix when the entries of $\mathbf{T}$ are all nonnegative.

2. A Hermitian matrix or a complex symmetric matrix when the entries of $\mathbf{T}$ are just real numbers(both positive and negative) or possibly complex.

This transformation is done in the following theorem:

Theorem 1 Given the tridiagonal matrix $T$ as in (3), and let $D_{1}=\operatorname{Diag}\left(\gamma_{1}, \gamma_{2}, \ldots, \gamma_{n}\right)$ be a diagonal matrix, where the sequence $\left\{\gamma_{i}\right\}_{i=1}^{n}$ is generated by: $\gamma_{n}=1$, and $\quad \gamma_{i}=$ $\sqrt{\frac{c_{i+1} c_{i+2} \ldots c_{n}}{b_{i} b_{i+1} \ldots b_{n-1}}} \cdot i=n-1, n-2, \ldots, 1$. Then the matrix $J=D_{1} \cdot T \cdot D_{1}^{-1}$ is the symmetric tridiagonal matrix:

$$
\mathbf{J}=\left[\begin{array}{ccccc}
a_{1} & \sqrt{b_{1} c_{2}} & 0 & \ldots & 0 \\
\sqrt{b_{1} c_{2}} & a_{2} & \sqrt{b_{2} c_{3}} & 0 & 0 \\
0 & \sqrt{b_{2} c_{3}} & a_{3} & \ldots & \\
& \cdots & & & \sqrt{b_{n-1} c_{n}} \\
& & 0 & \sqrt{b_{n-1} c_{n}} & a_{n}
\end{array}\right]
$$


where $\mathbf{J}$ is a tridiagonal matrix, and $\mathbf{J}$ is one of the following:

1. Either a real symmetric matrix,

2. Or a Hermitian matrix,

3. Or a complex symmetric matrix.

Proof. See [3].

Remark 2 The matrices $T$ and $\mathbf{J}$ have the same eigenvalues, $x_{1}, x_{2}, \ldots, x_{n}$, because they are similar.

Remark 3 The eigenvector of the matrix $\mathbf{J}$ that corresponds to the eigenvalue $x_{j}$ is: $\left[\gamma_{1} p_{0}\left(x_{j}\right), \gamma_{2} p_{1}\left(x_{j}\right), \ldots, \gamma_{n-1} p_{n-1}\left(x_{j}\right)\right]^{T}$.

\subsection{The Case of a Real Symmetric Matrix}

Given the $n \times n$ tridiagonal matrix $T$ as in (6), we use the recursion relation in (2) in order to generate the orthogonal polynomials $p_{0}(x), p_{1}(x), \ldots, p_{n-1}(x), p_{n}(x)$. Then a rootfinding method is used in order to compute the $n$ distinct real roots of $p_{n}(x)$. If the roots are $x_{1}, x_{2}, \ldots, x_{n}$, then these are the eigenvalues of $T$, and the corresponding eigenvectors are $\mathbf{P}_{j}=\left[p_{0}\left(x_{j}\right), p_{1}\left(x_{j}\right), \ldots, p_{n-1}\left(x_{j}\right)\right]^{T}, j=1,2, \ldots, n$. The next step is to fill in the columns of the orthogonal matrix $\mathbf{U}$ - That is $\mathbf{U}^{-1}=\mathbf{U}^{T}$-, and these columns are assigned the normalized vectors, $\frac{\left[\gamma_{1} p_{0}\left(x_{j}\right), \gamma_{2} p_{1}\left(x_{j}\right), \ldots, \gamma_{n-1} p_{n-1}\left(x_{j}\right)\right]^{T}}{N_{j}}$, where $N_{j}=\sqrt{\sum_{i=1}^{n} \gamma_{i}^{2} p_{i-1}^{2}\left(x_{j}\right)}, j=1, \ldots, n$.

Since $\mathbf{T}=\mathbf{D}_{1}^{-1} . \mathbf{J} . \mathbf{D}_{1}$, and $\mathbf{J}=\mathbf{U} . \mathbf{D}_{2} \cdot \mathbf{U}^{T}$, where $\mathbf{D}_{2}=\mathbf{D i a g}\left(x_{1}, x_{2}, \ldots, x_{n}\right)$, we have:

$$
\mathbf{T}=\left(\mathbf{D}_{1}^{-1} \mathbf{U}\right) \cdot \mathbf{D}_{2} \cdot\left(\mathbf{U}^{T} \mathbf{D}_{1}\right)
$$

Thus,

$$
\mathbf{T}^{2}=\left[\left(\mathbf{D}_{1}^{-1} \mathbf{U}\right) \cdot \mathbf{D}_{2} \cdot\left(\mathbf{U}^{T} \mathbf{D}_{1}\right)\right] \cdot\left[\left(\mathbf{D}_{1}^{-1} \mathbf{U}\right) \cdot \mathbf{D}_{2} \cdot\left(\mathbf{U}^{T} \mathbf{D}_{1}\right)\right]=\left(\mathbf{D}_{1}^{-1} \mathbf{U}\right) \cdot \mathbf{D}_{2}^{2} \cdot\left(\mathbf{U}^{T} \mathbf{D}_{1}\right)
$$

and

$$
\mathbf{T}^{k}=\left(\mathbf{D}_{1}^{-1} \mathbf{U}\right) \cdot \mathbf{D}_{2}^{k} \cdot\left(\mathbf{U}^{T} \mathbf{D}_{1}\right),
$$

where $k$ is any positive integer, and can be a negative integer when $\mathbf{T}$ is nonsingular. 


\subsection{The Case of a Hermitian Matrix}

This case is very similar to the case of a real symmetric matrix, in the sense that a Hermitian matrix has the same eigendecomposition which consists of a unitary matrix $\mathbf{U}$,structured the same way as the case of the orthogonal matrix in the above case, and it is known that the diagonal matrix $\mathbf{D}_{2}$ contains the eigenvalues of the Hermitian matrix, which are all just real numbers. So, the computation of powers of $\mathbf{T}$ follows almost the same procedure as the case of a real symmetric matrix.

\subsection{The Case of a Complex Symmetric Matrix}

Unforunately, there are not much references in the litrature that discuss the diagonalization of such matrices. One of the earliest articles is [6], which states that complex symmetric matrices can be diagonalized by a complex(orthogonal) transformation if and only if each eigenspace of the matrix has an orthonormal basis, which means that no eigenvectors have zero Euclidian norm are included in the basis, such eigenvectors are called quasi-null vectors, they are nonzero vectors but with zero norm, such as the vector $1+i$.

[14] discusses this for a special type of complex symmetric matrices, namely the matrices which have positive definite real and imaginary parts. [22] discusses the same case as well. The celebrated book of Horn and Johnson[15] gives an extended discussion of this subject in section (4.4, p201). This good book introduces the so-called Takagi factorization of complex symmetric matrices, then in further elaboration on the topic, a necessay and sufficient condition for a complex symmetric matrix A to be diagonalizable is introduced in Theorem 4.4.13, p211. This condition states that the matrix $\mathbf{A}$ must be orthogonally diagonalizable, that is $\mathbf{A}=\mathbf{S} \boldsymbol{\Lambda} \mathbf{S}^{-1}$, where $\boldsymbol{\Lambda}$ is diagonal, and $\mathbf{S}$ is nonsingular, and this is also equivalent to $\mathbf{A}=\mathbf{Q} \mathbf{\Lambda} \mathbf{Q}^{T}$, where $\mathbf{Q}$ is an orthogonal complex matrix, that is $\mathbf{Q}^{T}=\mathbf{Q}^{-1}$.

To make the discussion complete, we include here Takagi's factorization lemma-corollary 4.4.4, p204 in [15], and the theorem that gives necessary and sufficient conditions for a complex symmetric matrix to be diagonalizable- Theorem 4.4.13, p211 in [15].

Lemma 4 (Takagi's Factorization Lemma): Let $\mathbf{A} \in \mathbf{M}_{n}$ be symmetric, then there exists a unitary matrix $\mathbf{U} \in \mathbf{M}_{n}$ and a real nonnegative diagonal matrix $\mathbf{\Sigma}=\operatorname{diag}\left(\sigma_{1}, \sigma_{2}, \ldots, \sigma_{n}\right)$ such that $\mathbf{A}=\mathbf{U} \boldsymbol{\Sigma} \mathbf{U}^{T}$. The columns of $\mathbf{U}$ are an orthonormal set of eigenvectors for $\mathbf{A} \overline{\mathbf{A}}$, , and the corresponding entries of $\boldsymbol{\Sigma}$ are the nonnegative square roots of the corresponding eigenvalues of $\mathbf{A} \overline{\mathbf{A}}$.

It is worth to mention at this point that Takagi factorization is a special singular value decomposition of the symmetric matrix, as a matter of fact, it represents a scaled SVD. For more details see $[5,15]$. 
Theorem 5 (Theorem 4.4.13 in [15]: Let $\mathbf{A} \in \mathbf{M}_{n}$ be a symmetric matrix. Then $\mathbf{A}$ is diagonalizable if and only if it is complex orthogonally diagonalizable, that is $\mathbf{A}=\mathbf{S} \mathbf{\Lambda} \mathbf{S}^{-1}$, for a diagonal $\mathbf{\Lambda} \in \mathbf{M}_{n}$ and a nonsingular $\mathbf{S} \in \mathbf{M}_{n}$, if and only if $\mathbf{A}=\mathbf{Q} \mathbf{A} \mathbf{Q}^{T}$, where $\mathbf{Q} \in \mathbf{M}_{n}$ and $\mathbf{Q}$ satisfies $\mathbf{Q}^{T} \mathbf{Q}=\mathbf{I}$.

It is clear that both the corollary and the theorem do lead to the same conclusion, in the sense that a complex symmetric matrix can be factorized into the form $\mathbf{A}=\mathbf{U} \mathbf{\Sigma} \mathbf{U}^{T}$, and whence we obtain this, we can compute any powers of $\mathbf{A}$ using (7) above.

\section{Examples}

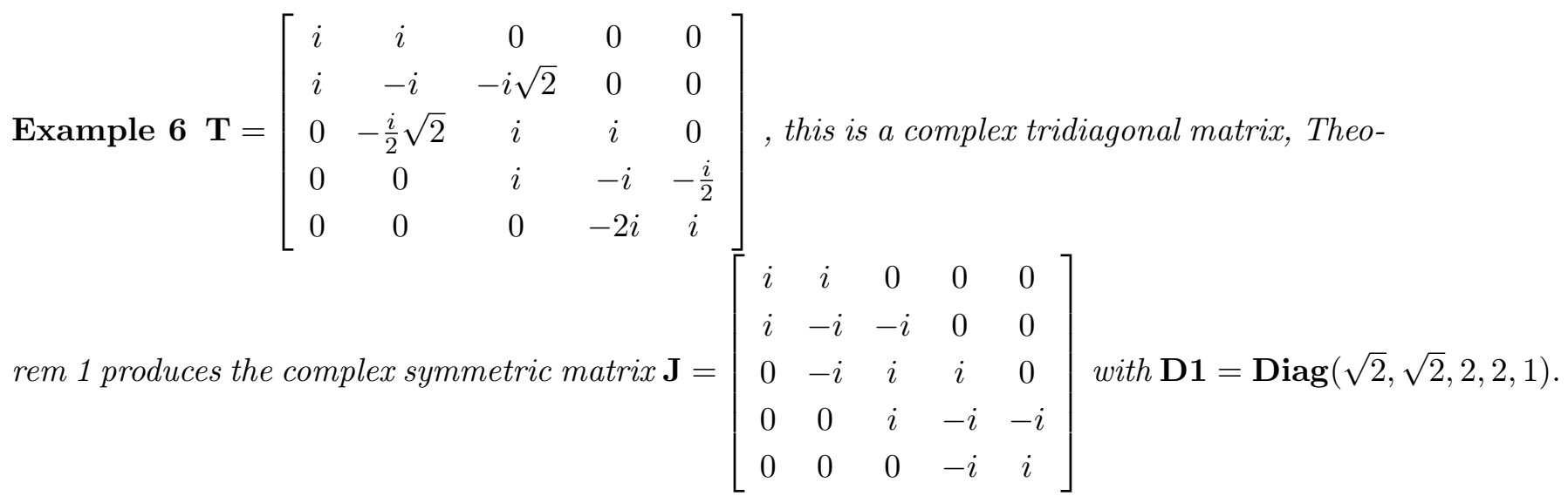

The matrix $\mathbf{D} 2=\operatorname{Diag}(\sqrt{2} i,-\sqrt{2} i, 2 i,-2 i, i)$. The normalized eigenvectors of $\mathbf{T}$ give the matrix

$$
\mathbf{U}=\left[\begin{array}{ccccc}
-0.6532814822 & -0.2705980499 & \frac{1}{2 \sqrt{2}} & \frac{1}{2 \sqrt{6}} & \frac{1}{\sqrt{3}} \\
-0.2705980499 & 0.6532814822 & \frac{1}{2 \sqrt{2}} & -\frac{\sqrt{6}}{4} & 0 \\
0 & 0 & -\frac{1}{\sqrt{2}} & -\frac{1}{\sqrt{6}} & \frac{1}{\sqrt{3}} \\
-0.2705980499 & 0.6532814822 & -\frac{1}{2 \sqrt{2}} & \frac{\sqrt{6}}{4} & 0 \\
0.6532814822 & 0.2705980499 & \frac{1}{2 \sqrt{2}} & \frac{1}{2 \sqrt{6}} & \frac{1}{\sqrt{3}}
\end{array}\right],
$$

Now, it is easy to compute $\mathbf{T}^{k}=\left(\mathbf{D} \mathbf{1}^{-1} \cdot \mathbf{U}\right) \cdot(\mathbf{D} 2)^{k} \cdot\left(\mathbf{U}^{T} \cdot \mathbf{D} 1\right)$,for any $k$.

Remark 7 Notice that this computation uses Theorem 4.4.13 in [15].

Example $8 \mathbf{T}=\left[\begin{array}{ccc}1 & -1 & 0 \\ -1 & 1 & 1 \\ 0 & 1 & 1\end{array}\right]$, this is a real symmetric matrix, so D1 is the identity matrix, and $\mathbf{D 2}=\operatorname{Diag}(\mathbf{1}, \mathbf{1}+\sqrt{2}, 1-\sqrt{2}), \mathbf{T} \cdot \overline{\mathbf{T}}=\left[\begin{array}{ccc}2 & -2 & -1 \\ -2 & 3 & 2 \\ -1 & 2 & 2\end{array}\right]$, the normalized eigenvectors of 
$\mathbf{T} \cdot \overline{\mathbf{T}}$ give the matrix $\mathbf{U}=\left[\begin{array}{ccc}\frac{1}{\sqrt{2}} & -\frac{1}{2} & -\frac{1}{2} \\ 0 & \frac{1}{\sqrt{2}} & -\frac{1}{\sqrt{2}} \\ \frac{1}{\sqrt{2}} & \frac{1}{2} & \frac{1}{2}\end{array}\right]$. Now, $\mathbf{T}^{k}=\mathbf{U} .(\mathbf{D} 2)^{k} . \mathbf{U}^{T}$, for any $k$

Remark 9 Notice that this computation uses the Takagi factorization lemma.

Example 10 This following example shows that the Takagi factorization is a scaled singular value decomposition.

$$
\mathbf{A}=\left[\begin{array}{ll}
1 & 2 \\
2 & 1
\end{array}\right] \text {, the eigenvalues of } \mathbf{A} \overline{\mathbf{A}} \text { are } 9 \text { and } 1 \text {, the SVD of } \mathbf{A}=\left[\begin{array}{cc}
\frac{1}{\sqrt{2}} & \frac{1}{\sqrt{2}} \\
\frac{1}{\sqrt{2}} & -\frac{1}{\sqrt{2}}
\end{array}\right]^{T}\left[\begin{array}{cc}
3 & 0 \\
0 & 1
\end{array}\right]\left[\begin{array}{cc}
\frac{1}{\sqrt{2}} & \frac{1}{\sqrt{2}} \\
-\frac{1}{\sqrt{2}} & \frac{1}{\sqrt{2}}
\end{array}\right],
$$

which can be scaled to give the Takagi factorization as follows: $\mathbf{A}=\left[\begin{array}{cc}\frac{1}{\sqrt{2}} & \frac{i}{\sqrt{2}} \\ \frac{1}{\sqrt{2}} & -\frac{i}{\sqrt{2}}\end{array}\right]\left[\begin{array}{ll}3 & 0 \\ 0 & 1\end{array}\right]\left[\begin{array}{cc}\frac{1}{\sqrt{2}} & \frac{1}{\sqrt{2}} \\ \frac{i}{\sqrt{2}} & -\frac{i}{\sqrt{2}}\end{array}\right]$, where the second column of $\mathbf{U}$ in the SVD is scaled by multiplication by the scalar $-i$.

\section{References}

1. Aiat Hadj, A. D., and Elouafi, M., A fast numerical algorithm for the inverse of a tridiagonal and pentadiagonal matrix, Applied Mathematics and Computation, vol. 202(2), 2008, pp 441-445.

http://dx.doi.org/10.1016/j.amc.2008.02.026

2. Al-Hassan, Q., On powers of tridiagonal matrices with nonnegative entries, Applied Mathemaical Sciences, Vol. 6, No. 48, 2012, pp2357-2368.

3. Al-Hassan, Q., Tridiagonal matrices and the computaion of Gaussian Quadratures, International J. of Pure and Applied Mathematics, Vol.55, No. 3, 2009, pp 301-310.

4. Al-Hassan, Q., An algorithm for computing inverses of tridiagonal matrices with applications, Soochow Journal of Mathematics, vo. 31, no. 3, 2005, pp 449-466.

5. Bunse-Gerstner, A., and Johnson, C. R., Singular value decomposition of complex symmetric matrices, J. of Computational and Applied Mathematics, Vol.21, 1988, pp41-54. http://dx.doi.org/10.1016/0377-0427(88)90386-x

6. Craven, B. D., Complex symmetric matrices, J. of Australian Mathematical Society, 10, 1969, pp341-354.

http://dx.doi.org/10.1017/s1446788700007588

7. Elouafi, M., and Aiat Hadj, A. D., On the powers and the inverse of a tridiagonal matrix, Applied Mathematics and Computation, vol. 211, 2009, pp 137-141. http://dx.doi.org/10.1016/j.amc.2009.01.026 
8. Elouafi, M., and Aiat Hadj, A. D., A new recursive algorithm for inverting Hessenberg matrices, Applied Mathematics and Computation, 214(2009), pp497-499.

http://dx.doi.org/10.1016/j.amc.2009.04.017

9. El- Mikkkawy, M., On the inverse of a general tridiagonal matrix, Applied Mathematics and Computation,150(2004), pp 669-679.

http://dx.doi.org/10.1016/s0096-3003(03)00298-4

10. El- Mikkkawy, M., and Karawia, A., Inversion of general tridiagonal matrices, Applied Mathematics Letters, 19(2006), pp712-720.

http://dx.doi.org/10.1016/j.aml.2005.11.012

11. El-Shehawey, M. A., El-Shreef, Gh. A., and Al-Henawy, A. Sh., Analytical inversion of general periodic tridiagonal matrices, Journal of Mathematical Analysis and Applications, 345(2008), pp123-134.

http://dx.doi.org/10.1016/j.jmaa.2008.04.002

12. Gutierrez-Gutierrez, J., Positive integer powers of certain tridiagonal matrices, Applied Mathematics and Computation, 202(2008), pp133-140.

http://dx.doi.org/10.1016/j.amc.2008.01.022

13. Gutierrez-Gutierrez, J., Powers of tridiagonal matrices with constant diagonals, Applied Mathematics and Computation, 206(2008), pp885-891.

14. Higham, N. J., Factoring complex symmetric matrices with positive definite real and imaginary parts, Mathematics of Computation, Vol. 67, No.224, 1998, pp1591-1599. http://dx.doi.org/10.1090/s0025-5718-98-00978-8

15. Horn, R. A., and Johnson, C. R., Matrix Analysis, Cambridge University Press, 1990.

16. Hou-Biao Li, Ting-Zhu Huang, Xing-Ping Liu, and Hong Li, On the inverses of general tridiagonal matrices, Linear Algebra and Its Applications, 433(2010), pp 965-983. http://dx.doi.org/10.1016/j.laa.2010.04.042

17. Kilic, Emrah, Explicit formula for the inverse of a tridiagonal matrix by backward continued fractions, Applied Mathematics and Computation, 197(2008), pp 345-357. http://dx.doi.org/10.1016/j.amc.2007.07.046

18. Rimas, Jonas, On computing of arbitrary positive integer powers for one type of symmetric tridiagonal matrices of even order-I, Applied Mathematics and Computation,168(2005), pp 783-787.

http://dx.doi.org/10.1016/j.amc.2004.09.017 
19. Rimas, Jonas, On computing of arbitrary positive integer powers for one type of symmetric tridiagonal matrices of odd order-I, Applied Mathematics and Computation,171(2005), pp 1214-1217.

http://dx.doi.org/10.1016/j.amc.2005.01.108

20. Rimas, Jonas, On computing of arbitrary positive integer powers for tridiagonal matrices with elements $1,0,0, \ldots, 0,1$ in principal and 1,1,..1 in neighbouring diagonals-II, Applied Mathematics and Computation, 187(2007), pp 1472-1475.

http://dx.doi.org/10.1016/j.amc.2006.09.078

21. Rimas, Jonas, On computing of positive integer powers for tridiagonal matrices with elements $-1,0,0, \ldots, 0,1$ in principal and 1,1,..1 in neighbouring diagonals-II, Applied Mathematics and Computation, 188(2007), pp 2020-2024.

http://dx.doi.org/10.1016/j.amc.2006.11.086

22. Serbin, S. M., On factoring a class of complex symmetric matrices without pivoting, Mathematics of Computation, Vol. 35, No. 152, 1980, pp1231-1234.

http://dx.doi.org/10.2307/2006388

Received: November 16, 2014; Published: January 9, 2015 